\title{
Current problems in assessing prognosis in heart failure: importance of biomarker patient's fingerprints
}

\author{
Azra Durak Nalbantic*, Mehmed Kulic, Nerma Resic, Enisa Hodzic, Mirza Dilic \\ Clinical Center University of Sarajevo, Sarajevo, Bosnia and Herzegovina
}

Heart failure (HF) is one of the most rapidly increasing cardiovascular disorder with unacceptably high mortality and readmissions rate. One possible solution to this problem could be the accurate assessment of prognosis of every single patient and personalized i.e. tailored therapeutic strategy, follow-up and allocation of resources according to the needs of each patient and thus improvement of the overall survival. But in real clinical practice we don't have clear recommendations how to adequately determine a prognosis. The situation has become more complex with recognition of HF with preserved ejection fraction; we don't know if we need separate prognostic model apart from HF with reduced EF. One possible reason for inadequate estimation of prognosis could be a fact that conventional predictors do not reflect newly discovered ongoing mechanisms in HF: inflammation, oxidative stress, neurohormonal activation, myocyte stress, injury and apoptosis, excessive or inadequate proliferation of the extracellular matrix.

Biomarkers released in the above mentioned processes could be potential new predictors of survival in HF. As it is used alone, single biomarker reflects only one ongoing process, but when used in combination they may become helpful in estimating prognosis and selecting an appropriate therapy. So the next logical step would be clustering biomarkers in multimarker panel in a way to optimise its use in assessing a prognosis. Multimarker approach could be a step toward tailored therapy for every patient based on the individual biomarker signal, i.e. biomarker patient's fingerprint. We hypothesized that combination of the next 4 bio- markers reflecting different pathological pathways in HF would improve prediction of survival: 1. BNP (myocytes stress), 2. troponin (myocytes injury), 3. cystatin C (renal function, ventricular remodelling and excessive interstitial fibrosis) and 4. tumor marker CA 125 (congestion and inflammation). It would be an interesting topic of future studies to see if the applied therapy could change the patient's biomarker fingerprint. Also there is an idea that the integration of different echocardiographic variables and biomarkers could have incremental, additive predictive value in estimating survival.

Conclusion: The combination of novel biomarkers in HF and echocardiographic parameters beyond left ventricle could play an important role in the prognosis and treatment of patients and may have a potential to reduce readmission rates and mortality.

KEYWORDS: heart failure, biomarkers, prognosis.

CITATION: Cardiol Croat. 2013;9(8):287.

\section{Received: $31^{\text {st }}$ Jul 2013}

*Address for correspondence: Klinički centar Univerziteta u Sarajevu, Bolnička 25, 71000 Sarajevo, Bosnia and Herzegovina.

Phone: +387-61-221-199

E-mail: azradurak@yahoo.com

\section{Literature}

1. Gheorghiade M, Vaduganathan M, Fonarow GC, Bonow RO. Rehospitalization for heart failure: problems and perspectives. J Am Coll Cardiol. 2013;61:391-403.

2. Braunwald E. Heart failure. JACC Heart Failure. 2013;1(1):1-20.

3. Zaya M, Phan A, Schwarz ER. Predictors of re-hospitalization in patients with chronic heart failure. World J Cardiol. 2012;4(2):23-30. 